\title{
Cholesterol-Induced Buckling in Physisorbed Polymer-Tethered Lipid Monolayers
}

\author{
Noor F. Hussain ${ }^{1}$, Amanda P. Siegel ${ }^{1,2}$, Merrell A. Johnson ${ }^{3}$ and Christoph A. Naumann ${ }^{1, *}$
}

1 Department of Chemistry and Chemical Biology, Indiana University-Purdue University Indianapolis, 402 N Blackford St., Indianapolis, IN 46202, USA; E-Mails: nfhussai@iupui.edu (N.F.H.); apsiegel@iupui.edu (A.P.S.)

2 Department Cellular and Integrative Physiology, Indiana University School of Medicine, Indianapolis, IN 46202, USA

3 Department of Physics, Indiana University_Purdue University Indianapolis, 402 N Blackford St., Indianapolis, IN 46202, USA; E-Mail: mermejoh@iupui.edu

* Author to whom correspondence should be addressed; E-Mail: canauman@iupui.edu; Tel.: +1-317-278-2512.

Received: 29 March 2013 / Accepted: 18 April 2013 / Published: 26 April 2013

\begin{abstract}
The influence of cholesterol concentration on the formation of buckling structures is studied in a physisorbed polymer-tethered lipid monolayer system using epifluorescence microscopy (EPI) and atomic force microscopy (AFM). The monolayer system, built using the Langmuir-Blodgett (LB) technique, consists of $3 \mathrm{~mol} \%$ poly(ethylene glycol) (PEG) lipopolymers and various concentrations of the phospholipid, 1-stearoyl-2-oleoyl-sn-glycero-3-phosphocholine (SOPC), and cholesterol (CHOL). In the absence of CHOL, AFM micrographs show only occasional buckling structures, which is caused by the presence of the lipopolymers in the monolayer. In contrast, a gradual increase of CHOL concentration in the range of $0-40 \mathrm{~mol} \%$ leads to fascinating film stress relaxation phenomena in the form of enhanced membrane buckling. Buckling structures are moderately deficient in CHOL, but do not cause any notable phospholipid-lipopolymer phase separation. Our experiments demonstrate that membrane buckling in physisorbed polymer-tethered membranes can be controlled through CHOL-mediated adjustment of membrane elastic properties. They further show that CHOL may have a notable impact on molecular confinement in the presence of crowding agents, such as lipopolymers. Our results are significant, because they offer an intriguing prospective on the role of CHOL on the material properties in complex membrane architecture.
\end{abstract}


Keywords: cholesterol; membrane elasticity; polymer-tethered lipid membrane; AFM; biaxial stress; buckling delamination; bilayer compartmentalization

\section{Introduction}

Buckling and wrinkling of thin elastic materials in response to lateral stress represents a fascinating topic of materials science and is of great significance in various technical applications $[1,2]$ Importantly, these stress relaxation processes are critically dependent on the stiffness of the underlying substrate [3]. On compliant substrates, thin elastic films respond to applied lateral stress through wrinkling and corresponding deformation of the substrate. In contrast, on stiff, non-compliant substrates, thin film buckling is characterized by buckle-delamination of distinct buckling amplitude, $w_{\max }$, and buckling width, $2 b$. In such cases, buckles often can be modeled as straight-sided blisters, for which the buckling theory of an Euler column can be applied. The Euler approach provides an analytical solution and links buckling parameters, such as $w_{\max }$ and $b$, with a plan strain modulus, bending modulus and film thickness [4,5].

Buckling and wrinkling phenomena can also be observed in biological membranes. Prominent examples include the lung surfactant system and the plasma membrane of phagocyting cells [6-10]. Because the complex architecture of biological membranes complicates a thorough analysis of buckling/wrinkling processes, efforts have been made to investigate such processes using model membrane systems. Simple lipid monolayer and bilayer systems are not well suited for such studies, because they are softer than typical biological membranes, whose elastic properties depend critically on the presence of embedded membrane proteins and presence of the membrane-linked cytoskeleton/extracellular matrix [11]. Previously, lipid monolayers with lung surfactant proteins have been successfully utilized to explore biophysical mechanisms associated with the reversible lipid monolayer collapse during the breathing cycle of a lung [8,12-14]. Recently, our group reported that physisorbed polymer-tethered membranes containing phospholipids, and lipopolymers represent another model membrane system, in which membrane buckling phenomena can be investigated under well-controlled conditions $[15,16]$. In this model system, the elastic properties can be modified simply through adjustment of lipopolymer molar concentration in the membrane $[17,18]$. For example, we showed that lipopolymer-phospholipid mixed monolayers physisorbed on a glass substrate following Langmuir-Blodgett (LB) film deposition show buckle-driven delamination at elevated lipopolymer molar concentration, leading to highly reproducible buckling patterns [15]. These patterns are visible by AFM, as the buckles have increased height and greater stiffness than the surrounding monolayer, and also by EPI with the use of dye-labeled lipids, as the buckled regions have an accumulation of lipids relative to the surrounding monolayer. Interestingly, in the presence of poly(2-ethyl-2-oxazoline) and poly(ethylene glycol) (PEG) lipopolymers, buckling regions in the LB monolayer prevented the formation of a phospholipid bilayer through Langmuir-Schaefer (LS) transfer [15]. Here, the bilayer compartmentalization was attributed to a stress relaxation effect, which is associated with the partial penetration of polymer moieties of lipopolymers into the hydrophobic region of the bottom monolayer, thus preventing bilayer formation at buckling regions. More recently, we also derived a metric between 
membrane elasticity and quantifiable buckling parameters, $w_{\max }$ and $b$, in physisorbed mixed monolayers of poly(ethylene glycol) (PEG) lipopolymers and phospholipids by combining mean-field calculations of polymer-tethered membranes and buckling theory for a straight-sided blister [16]. Notably, physisorbed polymer-tethered lipid bilayers, which are compartmentalized by buckling structures, show fascinating length scale-dependent lipid diffusion properties with remarkable parallels to those found in plasma membranes [19]. These dynamic properties are also intriguing in light of the recently reported fabrication of such membrane systems with either a static lipopolymer concentration gradient or sharp boundaries between regions of low and high lipopolymer concentrations [20].

Buckling theory predicts a relationship between buckling structure parameters, such as buckling width, $2 b$, and buckling amplitude, $w_{\max }$, and bending modulus, $K_{\mathrm{c}}$, of the thin film[5]. In the current paper, we demonstrate that membrane buckling properties can also be altered by varying $K_{\mathrm{c}}$ of the physisorbed polymer-tethered membrane through variation of cholesterol (CHOL) molar concentration. In particular, we investigate the influence of CHOL molar concentration on the buckling behavior in membranes under conditions of comparably low lipopolymer concentrations of $3 \mathrm{~mol} \%$ 1,2-distearoyl-sn-glycero-3-phosphoethanolamine- $n$-[methoxy(polyethylene glycol)-5000] (DSPE-PEG 5000). At these DSPE-PEG5000 molar concentrations, physisorbed polymer-tethered lipid bilayers lack substantial buckling in the absence of CHOL. This experimental strategy was pursued because CHOL content is well known to influence bending elasticity in model and biological membranes [21-26]. The current work is intriguing in light of the important role of CHOL on membrane elasticity and functionality in biological membranes.

\section{Experimental Section}

\subsection{Materials}

The phospholipids, 1-stearoyl-2-oleoyl-sn-glycerol-3-phosphocholine (SOPC) and cholesterol (CHOL), and the lipopolymer, 1,2-distearoyl-sn-glycero-3-phosphoethanol amine- $n$-[methoxy (polyethylene glycol)-5000] (DSPE-PEG 5000), were purchased from Avanti Polar lipids (Alabaster, AL, USA). The dye-labeled cholesterol 5-cholesten-3ß-ol-6-[(7-nitro-2-1,3-benzoxadiazol-4-yl) amino]caproate (NBD-6-cholesterol) was obtained from Avanti Polar Lipids (Alabaster, AL). The fluorescently labeled phospholipid, $N$-(6-tetramethylrhodamine-thiocarbamoyl)-1,2-dihexadecanoylsn-glycer-3-phosphoethanolamine, triethylammonium salt (TRITC-DHPE), was purchased from

Invitrogen (Eugene, OR, USA). The dye-labeled lipopolymer 1,2-dipalmitoyl-sn-glycero-3phosphatidylethanolamine- $N$-[amino(polyethylene glycol)2000]-TAMRA (DPPE-PEG2000-TAMRA) was synthesized from the sodium salt of the amino-functionalized lipopolymer (Avanti Polar Lipids, Alabaster, AL, USA) and TAMRA (Invitrogen, Eugene, OR, USA), as described before [27]. Chloroform (HPLC grade, Fisher Scientific, Pittsburgh, PA, USA) was used as a spreading solvent to form monolayers at the air-water interface. The ultrapure water (Milli-Q) was obtained from Millipore Water Purification System (Milford, MA, USA). Glass coverslips (24 mm $\times 40 \mathrm{~mm}$, No. 1) were purchased from VWR Scientific Products (West Chester, PA, USA). Glass coverslips were cleaned before usage, as described previously [16]. 


\subsection{Preparation of Polymer-Tethered Phospholipid Monolayers and Bilayers}

Physisorbed polymer-tethered phospholipid monolayers and bilayers were prepared following procedures described before [21]. Physisorbed monolayer systems were built using the Langmuir Blodgett (LB) technique. Here, the LB monolayer was prepared first by spreading a mixture of phospholipids, cholesterol and lipopolymers [compositions: 3 mol \% DSPE-PEG 5000, 0-40 mol \% CHOL, 56.5-96.5 mol \% SOPC, $0.5 \mathrm{~mol} \%$ TRITC-DHPE) in chloroform at the air-water interface of a film balance with dipper. (Labcon, Darlington, UK). The mixed monolayer was compressed to a film pressure of $30 \mathrm{mN} / \mathrm{m}$ and, after an equilibration time of $25 \mathrm{~min}$, transferred to glass slides (VWR cover glass No. 1: $24 \mathrm{~mm} \times 40 \mathrm{~mm}$ ), mounted on the dipper. The LB transfer was conducted using a dipping speed of $5 \mathrm{~mm} / \mathrm{s}$. Monolayer samples were used for EPI and atomic force microscopy (AFM) analyses within $24 \mathrm{~h}$. To obtain physisorbed polymer-tethered bilayers, a second monolayer comprised of phospholipids and cholesterol (compositions: SOPC, 0-40 mol \% CHOL, $0.5 \mathrm{~mol} \%$ TRITC-DHPE) was added to the LB monolayer using Langmuir-Schaefer (LS) deposition. To conduct LS transfers, a depression slide was placed at the floor of the film balance. Next, the LB substrate with the monolayer was gently, but forcefully pushed into the glass coverslip containing the monolayer and placed on the depression slide. Bilayer samples were analyzed within $4 \mathrm{~h}$ after completion of their assembly. All imaging experiments on polymer-tethered monolayer systems were conducted in air. Corresponding bilayer studies were pursued in Millipore water.

\subsection{Epifluorescence Microscopy and Analysis}

Epifluorescence microscopy images were taken using an inverted optical microscope (Axiovert 200M, Zeiss, Oberkochen, Germany) equipped with an AxioCam MRm monochrome digital camera [15]. The microscope was operated in epi-illumination, in which the beam was focused to the sample by a microscopy objective (Zeiss C-Apochromat, water immersion, $40 \times \mathrm{NA}=1.2$ ) with optional Optovar magnification (1.6×). EPI images were acquired and analyzed using Axiovision software (Zeiss, Oberkochen, Germany) and ImageJ (National Institutes of Health). To obtain information about the bearing area (BA), EPI images were analyzed using the area percentage analysis method in the ImageJ software. Specifically, for each opened image, the foreground and background colors were set to white and black, respectively, by using the "edit" options and color tool bars. Next, we utilized the "set measurements" option from the analyze tool bar, selected the area of interest, adjusted the threshold and determined the bearing area on the basis of the brightness of the image.

\subsection{Atomic Force Microscopy and Image Analysis from Nanoscope 6.1 Software}

AFM experiments were conducted on glass-supported polymer-tethered lipid monolayers using a digital Instruments Bio-Scope (Digital Instruments/Vecco Metrology group, Plainview, NY, USA). AFM micrographs were analyzed using Nanoscope IV (V6.12) (Digital Instruments/Vecco Metrology group, Plainview, NY, USA) and Origin 8 (Origin lab Corporation, Northampton, MA, USA). AFM data were acquired in soft tapping mode using silicon nitride AFM probes (Budget sensors, Innovative solutions Bulgaria Ltd., Sofia, Bulgaria) with a spring constant of $\sim 0.27 \mathrm{~N} / \mathrm{m}$ and a tip radius of $<15 \mathrm{~nm}$. Image sizes of $10 \times 10 \mu \mathrm{m}^{2}$ and $5 \times 5 \mu \mathrm{m}^{2}$ sections were taken with a scan rate of $1 \mathrm{~Hz}$ and 
with a resolution of $256 \times 256$ pixels. AFM images were taken within $24 \mathrm{~h}$ of the preparation of the monolayers. All AFM data were analyzed using the section analysis tool to determine the width and height of buckling structures. The AFM section analysis was pursued at approximately half-micron intervals in the $40 \%$ cholesterol containing monolayers to obtain the buckle height and the buckle width. In monolayers with less than $40 \%$ cholesterol, the section analysis was done on the buckles, which are above the height of $2 \mathrm{~nm}$. In order to achieve statistical significance, values of average buckle width and buckle height and associated standard deviations were determined from about 60 different section analyses out of three separate AFM images per sample. In this case, the buckling width was identified from the intersections of line fits of the shoulders of buckling peaks and the base around the buckle of interest. The mean buckling width was obtained by examining the distribution of buckling widths from multiple sectional profile analyses. Buckling height values were determined from the difference between peak height and line fit of the baseline around the buckle. Again, the average buckle height was acquired from the distribution of buckling heights from multiple section analyses.

\section{Results and Discussion}

Physisorbed polymer-tethered lipid bilayers comprised of phospholipids and lipopolymers represent model membrane systems with fascinating tunable elastic properties. Importantly, their membrane elasticity can be adjusted by the molar concentration of lipopolymers in the bottom leaflet of the bilayer [16]. As reported before, the presence of elevated concentrations of membrane stress-imparting lipopolymers may lead to a stress relaxation processes, such as buckling delamination of the membrane on solid substrates [15]. At low lipopolymer concentrations (low lateral stress), polymer-tethered monolayers and bilayers typically lack substantial buckling formation. In contrast, at medium to high lipopolymer content (high lateral stress), buckling structures can be observed, which become more prominent in terms of buckling width and bearing area (percentage of buckling region), with increasing lipopolymer molar concentration [16]. Interestingly, the EPI micrographs in Figure 1A-C demonstrate that membrane buckling in polymer-tethered membranes can also be caused by varying concentrations of CHOL. In this case, well-developed buckling structures can be resolved by optical microscopy at $40 \mathrm{~mol} \% \mathrm{CHOL}$ (represented by the lighter structures in $\mathrm{C}$ ), but not between 5 (A) and $30 \mathrm{~mol} \%$ CHOL (B), which show buckling blisters, but not connected structures. The presence of buckling structures in Figure 1C is remarkable, because the monolayer only contains 3 mol \% DSPE-PEG 5000, which without CHOL, does not cause any sizable membrane buckling [16].

To analyze the buckling structures in more detail, Figure $2 \mathrm{~A}-\mathrm{C}$ presents AFM micrographs of polymer-tethered monolayers of comparable compositions. At $5 \mathrm{~mol} \% \mathrm{CHOL}$, occasional circular blisters can be observed (Figure 2A). At $30 \mathrm{~mol} \% \mathrm{CHOL}$, the monolayer is characterized by a substantially increased density of circular blisters (Figure 2B). Further increase of CHOL molar concentration to $40 \mathrm{~mol} \%$ leads to the formation of ridge-like buckling structures with junctions (Figure 2C), which mirror those from EPI experiments in Figure 1C. In contrast, no significant buckling can be observed on a monolayer system with 40 mol \% CHOL, but without DSPE-PEG 5000 (Figure 2D). 
Figure 1. Epifluorescence microscopy (EPI) micrographs of physisorbed polymer-tethered lipid monolayers comprised of 1-stearoyl-2-oleoyl-sn-glycero-3-phosphocholine (SOPC), $3 \mathrm{~mol} \%$ 1,2-distearoyl-sn-glycero-3-phosphoethanol amine- $n$-[methoxy(polyethylene glycol)-5000] (DSPE-PEG 5000) and $5 \mathrm{~mol} \%$ (A), $30 \mathrm{~mol} \mathrm{\%} \mathrm{(B)} \mathrm{and} 40 \mathrm{~mol} \%$ cholesterol (CHOL) (C). To conduct EPI experiments, each monolayer also contains $0.5 \% \mathrm{~N}$-(6tetramethylrhodamine-thiocarbamoyl)-1,2-dihexadecanoyl-sn-glycer-3-phosphoethanolamine, triethylammonium salt (TRITC-DHPE). The white scale bar represents $10 \mu \mathrm{m}$ in size.
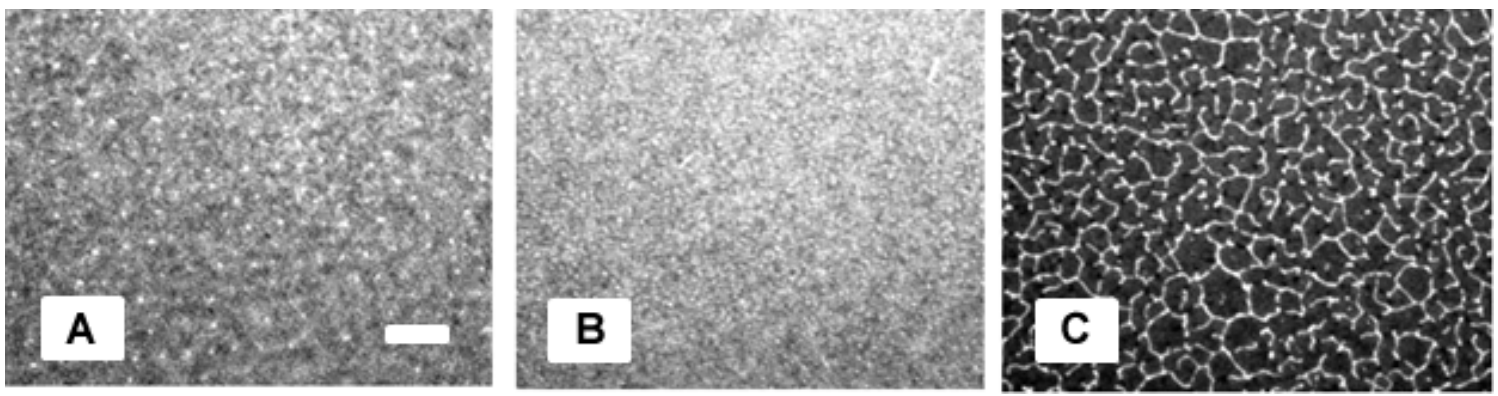

This indicates that at least small amounts of lipopolymers are required for sizable membrane buckling to occur. The observed EPI and AFM micrographs in Figures 1 and 2 are significant, because they confirm that variations in CHOL molar concentrations, which modify membrane bending elasticity, are associated with changes in membrane buckling behavior. Notably, they show intriguing parallels to recent experiments on comparable membrane systems, in which buckling structures were altered by changing lipopolymer molar concentrations $[15,16]$. These intriguing parallels suggest a mechanism in which enhanced membrane buckling can be seen as a stress relaxation phenomenon in response to increasing film stress affecting molecular confinement in the model membrane.

Figure 2. Atomic force microscopy (AFM) micrographs of polymer-tethered lipid monolayers containing SOPC, 3 mol \% DSPE-PEG 5000 and 5 mol \% (A), 30 mol \% (B) and $40 \mathrm{~mol} \% \mathrm{CHOL}(\mathbf{C})$. For comparison, an AFM micrograph from a SOPC monolayer with $40 \mathrm{~mol} \% \mathrm{CHOL}$, but without DSPE-PEG5000, is shown as well (D). The image size is $10 \mu \mathrm{m} \times 10 \mu \mathrm{m}$.

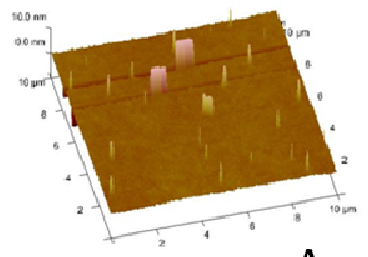

A

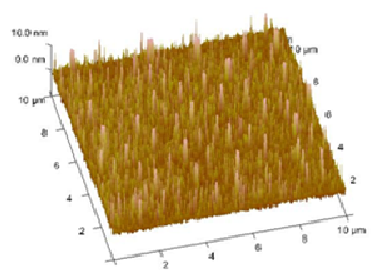

B

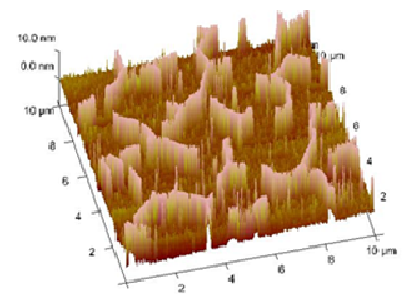

C

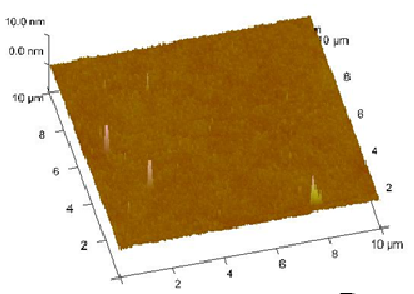

D

Scaling arguments of polymer physics suggest that the polymer moiety of 3 mol \% DSPE-PEG5000 is close to the mushroom-brush transition. Under these conditions, lipopolymers, which consist of a bulky polymer moiety and a comparable small lipid anchor, primarily interact via repulsive polymer-polymer interactions, and phospholipids act as template molecules with good mixing properties. In this case, lipopolymer phase segregation, as assumed in a hypothetical thermodynamically-driven phase separation, is unlikely to occur. This is because such a segregation 
process would be associated with the energetically unfavorable stretching of polymer chains. However, partial cholesterol segregation in membranes with regions of different membrane curvatures cannot be excluded. To explore the possibility of such a phase segregation, Figure 3 compares the distribution of dye-labeled lipopolymers (A,C), phospholipids (B) and cholesterol (D) in a polymer-tethered lipid monolayer comprised of 3 mol \% DSPE-PEG 5000, $40 \mathrm{~mol} \%$ CHOL and $55.8 \mathrm{~mol} \%$ SOPC (dye molecule concentration: $0.6 \mathrm{~mol} \%$ ). Comparison of the DPPE-PEG2000-TAMRA (A) and NBD-DHPE (B) in the same region of the monolayer demonstrates that there is no clearly detectable lipopolymer-phospholipid phase separation in the monolayer, which agrees well with recent findings on comparable membrane systems without cholesterol (15). This result supports the notion of a largely homogeneous lipopolymer distribution in the polymer-tethered membrane with phospholipids acting as template molecules. In contrast, the EPI micrographs of the DPPE-PEG2000-TAMRA (C) and NBD-6-cholesterol (D) distributions show that buckling structures are partially depleted in cholesterol. This partial depletion of CHOL in buckling structures is plausible, because CHOL is known to prefer regions of low membrane curvature over those of higher membrane curvature.

Figure 3. EPI micrographs comparing the distribution of TAMRA-DSPE-PEG2000 (A) and NBD-DHPE (B), as well as TAMRA-DSPE-PEG2000 (C) and NBD-6-cholesterol (D) in a physisorbed polymer-tethered monolayer system consisting of $3 \mathrm{~mol} \%$ DSPE-PEG 5000, $40 \mathrm{~mol} \% \mathrm{CHOL}$ and $55.8 \mathrm{~mol} \%$ SOPC (dye molecule concentration: $0.6 \mathrm{~mol} \%$ ). The size of the scale bar is $10 \mu \mathrm{m}$.

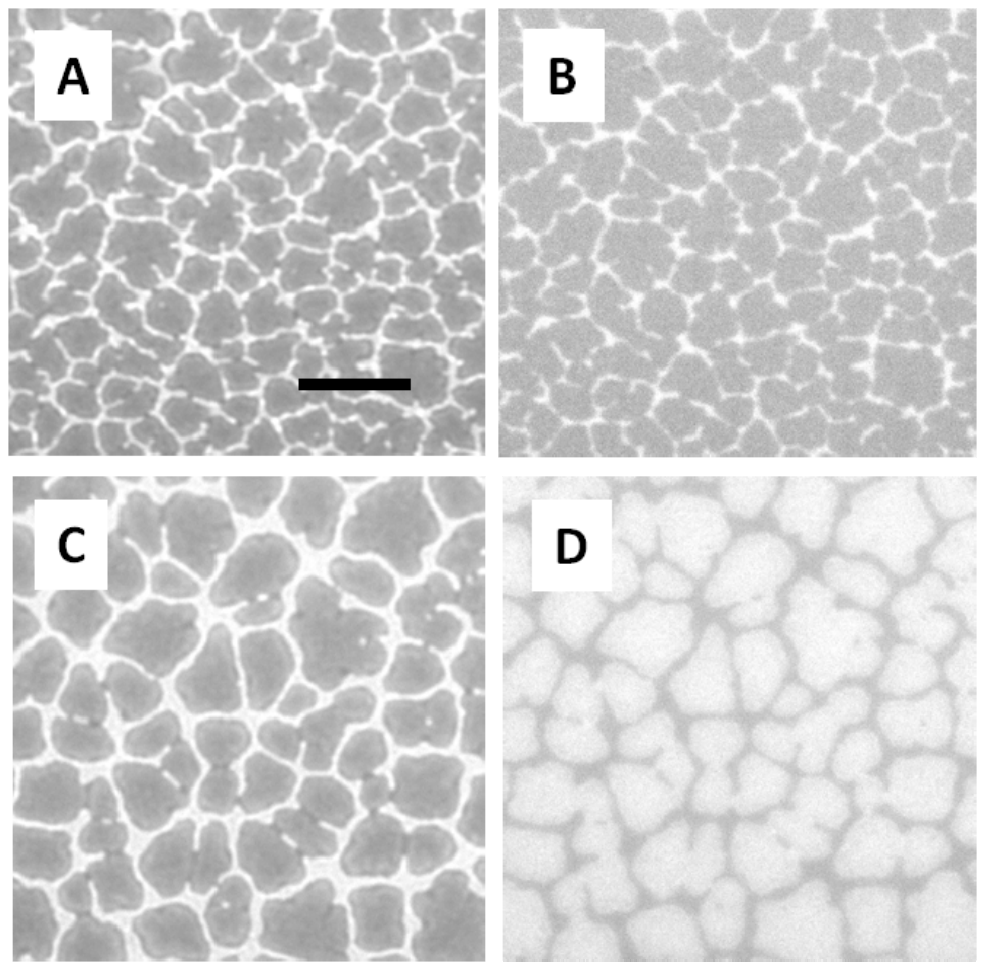

Figure 4 illustrates the influence of CHOL content on the membrane organization in physisorbed polymer-tethered lipid bilayers. Specifically, EPI micrographs of bilayer systems with 0 mol \% (A) and $40 \mathrm{~mol} \% \mathrm{CHOL}$ (B) are compared. The two micrographs show distinct heterogeneities, which reflect those of buckling structures on polymer-tethered monolayers determined by AFM in Figure 2. 
Comparison of the EPI micrographs obtained from monolayer and bilayer systems in Figures 1 and 4 demonstrates a phase inversion of the fluorescence signal, in excellent agreement with findings on corresponding polymer-tethered membranes with poly(2-ethyl-2-oxazoline) lipopolymers reported previously [15]. For example, the bright buckling regions with a darker background in Figure 1C become dark regions with a brighter background in Figure 4B. On the basis of AFM experiments on physisorbed polymer-tethered monolayer and bilayer systems, this phase inversion has been attributed to the inability of bilayer formation at buckling regions in the presence of lipopolymers with only moderately hydrophilic polymer moieties, such as PEG or poly(2-ethyl-2oxazoline) [15]. This interpretation was supported by control experiments on polymer-tethered membranes with more hydrophilic poly(2-methyl-2-oxazoline) lipopolymers, which showed buckling, but no prevention of bilayer formation at buckling regions. These findings suggest a stress relaxation process on PEG and poly(2-ethyl-2-oxazoline) lipopolymers, in which moderately hydrophilic polymer chains partially penetrate into the hydrophobic region of the delaminated LB monolayer. As a result of this polymer relaxation process, the surface of buckling regions becomes more hydrophilic, thus preventing the formation of the LS lipid monolayer at these regions. Importantly, the "buckling-induced dewetting" process leads to the formation of lipid diffusion barriers (inset of Figure 4), thus compartmentalizing the bilayer system. As discussed previously, the bilayer compartmentalization is associated with fascinating dynamic properties [15]. The regions of "buckling-induced dewetting" become efficient diffusion obstacles and/or diffusion boundaries. As recent wide-field single molecule tracking experiments on physisorbed polymer-tethered bilayers have shown, length scale-dependent lipid diffusion properties can be observed with remarkable parallels to those found in cellular membranes. In the latter case, membrane compartmentalization has been attributed to membrane-linkages of the cytoskeleton [22]. Previously, we pointed out that the permeability of buckling-associated bilayer boundaries can be regulated by adjustment of lipopolymer molar concentration in these model membranes [15]. Our current work suggests that such a regulation of permeability can also be accomplished by changing CHOL molar concentration in physisorbed polymer-tethered lipid bilayers.

Figure 4. EPI micrographs of physisorbed polymer-tethered lipid bilayer with 0 mol \% (A) and $40 \mathrm{~mol} \% \mathrm{CHOL}(\mathbf{B})$ in SOPC with $3 \mathrm{~mol} \%$ DSPE-PEG 5000 and $0.5 \mathrm{~mol} \%$ TRITC-DHPE (The size of the scale bar is $10 \mu \mathrm{m}$ ). The inset (size: $20 \mu \mathrm{m} \times 20 \mu \mathrm{m}$ ), which illustrates the boundary region of a bleaching spot, demonstrates that buckling regions act as lipid diffusion barriers, as reported previously [15].

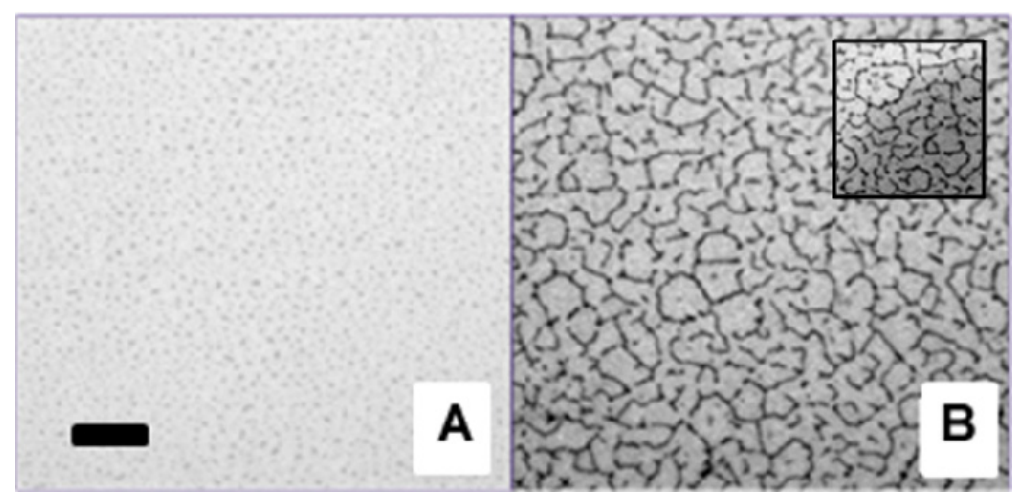


Figures 1-4 empirically show that CHOL content influences membrane buckling in polymer-tethered membranes containing low concentrations of lipopolymers. As reported recently, a quantitative relationship between buckling structure parameters (i.e., buckling amplitude, $w_{\max }$, and width, $b / 2$ ) and membrane elastic properties can be developed [16]. Here, the buckling structure parameters, $w_{\max }$ and $b$, can be obtained experimentally using AFM section and EPI fluorescence intensity analyses. Then, the buckling theory of an Euler column can be applied to link these buckling structure parameters to film elastic properties. The Euler column approximation is feasible, because the requirements of notable higher substrate (glass) stiffness relative to membrane stiffness [5] and of substantially larger values of buckling widths versus buckling heights [5] are fulfilled [16]. In the Euler column approach, the bending stiffness, $K_{\mathrm{c}}$, and film thickness, $h$, are related to the plane strain modulus, $E_{f}^{*}$, by [5]:

$$
K_{c}=\frac{E_{f}^{*} h^{3}}{12}
$$

Furthermore, the ratio of the maximum height of the buckles, $w_{\max }$, to the film thickness, $h$, is closely associated with the non-dimensional loading parameter, $\sigma_{\mathrm{o}} / \sigma_{\mathrm{c}}\left(\sigma_{\mathrm{o}}\right.$ : film stress, $\sigma_{\mathrm{c}}$ : critical stress at the onset of buckling [5].

$$
\left(\frac{w_{\max }}{h}\right)=\sqrt{\frac{4}{3}\left(\frac{\sigma_{o}}{\sigma_{c}}-1\right)}
$$

The critical stress can also be calculated independently from the bending stiffness and thickness of the film, as well as the buckling width, $b / 2$ and height of the buckles, $w_{\max }$, and from this, $\sigma_{\mathrm{o}}$ [5]:

$$
\sigma_{c}=\frac{\pi^{2} K_{c}}{b^{2} h}
$$

The bending modulus, $K_{\mathrm{c}}$, and thickness, $h$, of lipopolymer/lipid mixtures as a function of lipopolymer content, $X_{\mathrm{p}}$, can be derived from mean-field calculations of polymer-tethered membranes [23]. According to the mean-field approach, $K_{\mathrm{c}}$ of a lipopolymer/lipid mixture with $20 \mathrm{~mol} \%$ DSPE-PEG5000 is about $400 k_{\mathrm{B}} T$, which corresponds to a bending elasticity found in Dictyostelium discoideum (wild-type) [24]. In contrast, $K_{\mathrm{c}}$ of $5 \mathrm{~mol} \%$ DSPE-PEG5000 is about $50 k_{\mathrm{B}} T$, similar to values observed in red blood cells [25]. The film thickness, $h$, represents the sum of the thickness values of polymer and lipid moieties in the membrane. The thickness of the polymer part is assumed to remain constant at a value of $8.8 \mathrm{~nm}$ [17]. In contrast, the thickness of the lipid moiety is considered to increase by $20 \%$ in the range from 0 to $40 \mathrm{~mol} \% \mathrm{CHOL}$ with thickness values ranging from 2.5 to $3 \mathrm{~nm}$, as reported previously [26].

Figure 5 illustrates an example of the quantitative AFM section analysis of buckling structures in a physisorbed polymer-tethered lipid monolayer. The positions of the section analysis on the micrographs were selected manually using the section analysis tool of the AFM software (indicated as white line in Figure 5). The resulting surface profile is shown as an inset. The AFM section analysis was pursued at approximately half-micron intervals in the $40 \%$ cholesterol containing monolayers to obtain the buckle height and the buckle width. In monolayers with less than $40 \%$ cholesterol, the 
section analysis was done on all buckles above a threshold height of $2 \mathrm{~nm}$. In order to achieve statistical significance, three separate AFM images from each cholesterol category were used to get the height and the width of the buckles, and the mean and the standard deviation were calculated.

Figure 5. Section analysis of AFM micrograph obtained from physisorbed polymer-tethered lipid monolayer containing SOPC together with $40 \mathrm{~mol} \%$ CHOL and $3 \mathrm{~mol} \%$ DSPE-PEG5000. Image size: $10 \mu \mathrm{m} \times 10 \mu \mathrm{m}$. The inset shows the height profile along the line in the micrograph. It allows the quantitative analysis of buckling structures in terms of buckling amplitude and buckling width.

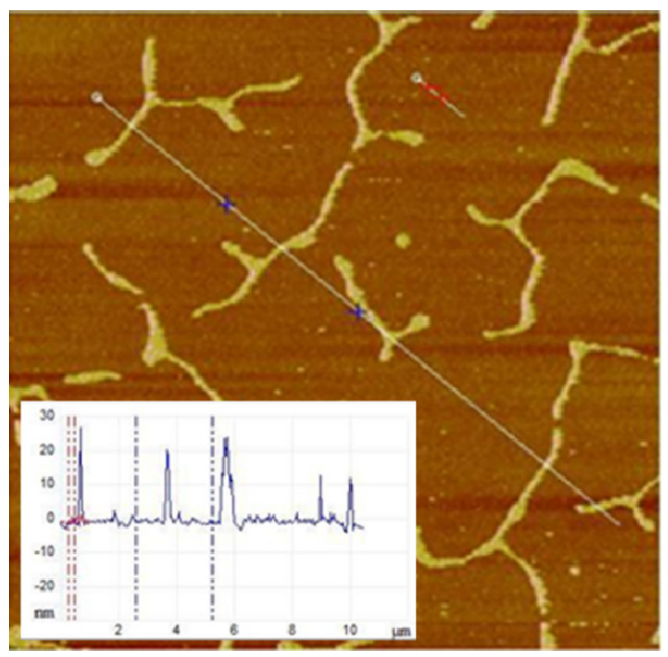

The quantitative link between buckling structures and membrane elastic properties is illustrated in Figure 6A,B. As Figure 6A shows, there is a linear relationship between bearing area, $B A$ and $\mathrm{CHOL}$ molar concentration in the physisorbed polymer-tethered lipid monolayer. Linear scaling between $B A$ and increased stiffness (as shown by increasing DSPE-PEG5000 content) was also observed on SOPC/DSPE-PEG 5000 mixed monolayers (without $\mathrm{CHOL}$ ) in the range of $0-20 \mathrm{~mol} \%$ lipopolymer [16]. However, this system did not show any significant change in $B A$ for lipopolymer molar concentrations of more than $20 \mathrm{~mol} \%$ DSPE-PEG5000. Figure $6 \mathrm{~B}$ exhibits that that there is also a linear relationship between film stress, $\sigma_{\mathrm{o}}$ and $\mathrm{CHOL}$ molar concentration.

Figure 6. Impact of CHOL molar concentration on bearing area (percentage of buckling regions) (A) and biaxial stress, $\sigma_{\mathrm{o}},(\mathbf{B})$ in a physisorbed polymer-tethered lipid monolayer, as obtained from analysis of EPI and AFM micrographs.
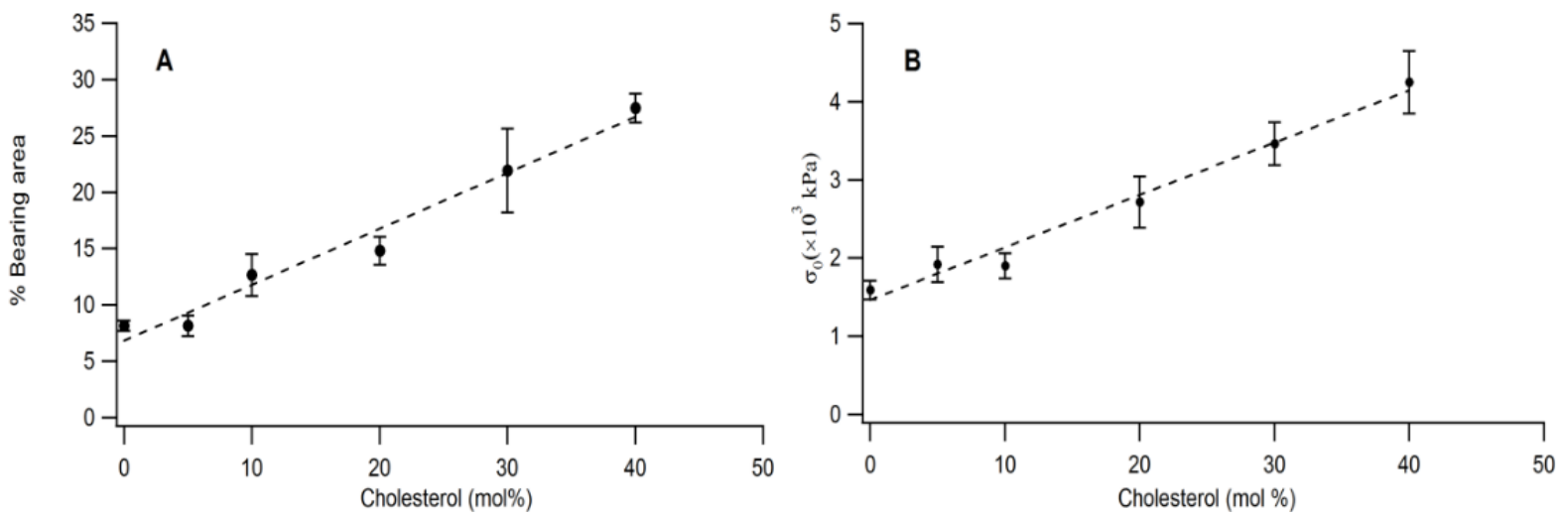
Together, our data demonstrate that there is a strong correlation between CHOL molar concentration, $B A$ and $\sigma_{0}$. This result is significant, because it shows that CHOL content in polymer-tethered membranes not only influences membrane bending elasticity, but also membrane buckling behavior. Comparison to previous results on physisorbed polymer-tethered monolayers of different lipopolymer concentrations reveals that changes in CHOL and lipopolymer concentration demonstrate a qualitatively similar impact on membrane elasticity and buckling $[15,16]$. The observed CHOL-mediated membrane buckling can be understood in light of the general knowledge about stress relaxation phenomena of lipopolymers in polymer-tethered membranes and the impact of CHOL on lipid packing and membrane stiffness. To reduce the lateral stress caused by repulsive interactions of their polymer chains, lipopolymers are known to cause different stress relaxation phenomena, such as membrane roughening (outside buckling regions) and partial penetration of moderately hydrophilic polymer chains of lipopolymers into the lipid region of the membrane [15,27]. Cholesterol addition is expected to counter these stress relaxation effects by increasing lipid packing density and membrane stiffness. Indeed, analysis of membrane micro-roughness outside buckling areas over a wide range of CHOL concentrations supports such a process of membrane flattening (data not shown). In other words, the partial suppression of lipopolymer-induced membrane stress relaxation will lead to an increase of repulsive polymer-polymer interactions of lipopolymers, which is associated with an enhancement of lateral stress in the membrane.

Our current findings are intriguing in light of the important functional role of CHOL in biological membranes $[28,29]$. The influence of CHOL on membrane elastic properties, such as membrane bending and compressibility, is well documented [29,30]. Less investigated, however, is the role of CHOL in membrane buckling. Previously, it has been pointed out that CHOL co-localizes with curved membrane structures, such as clathrin-coated pits, caveolae and synaptic vesicles, thereby influencing membrane protein sequestration [31-33]. Furthermore, cytoskeletal assembly and disassembly appears to be closely associated with changes of membrane shape [34,35]. CHOL presumably plays a role in such shape changes, as the degree of cytoskeleton-membrane adhesion in plasma membranes was reported to depend on CHOL concentration in the membrane [36]. CHOL was also observed to be critically involved in the reversible wrinkling and folding of the membrane during the normal breathing cycle in the lung. For example, Bernardino and co-workers reported on the significance of $\mathrm{CHOL}$ in the structure and function of lung surfactants [37]. They showed that CHOL helps to create defined lateral structures in lung surfactant bilayers at normal physiological temperatures. Such bilayers comprised of a lipid mixture and lung surfactants are able to withstand the rapid change of interfacial equilibrium pressure during breathing.

Our AFM data in Figure 2 demonstrate that simple phospholipid-CHOL mixtures typically do not form stable buckling structures without lipopolymers. This suggests that the presence and enrichment of cone-shaped lipopolymers (lipopolymers have a bulky polymer moiety and a comparably small lipid anchor) is crucial for the buckling process to occur. In other words, membrane buckling can be seen as a stress relaxation phenomenon caused by molecular confinement of cone-shaped molecules, like lipopolymers, which impose a lateral stress on the model membrane [15]. Interestingly, steric confinement of certain proteins in membranes can also induce membrane curvature [38]. These remarkable parallels suggest that lipopolymers behave like crowding agents, which not only lead to molecular confinement, but also possibly cause changes of membrane shape. Processes of molecular 
confinement leading to alterations in membrane shape are potentially significant, because multiple membrane-associated processes are known to respond sensitively to membrane curvature [39].

\section{Conclusions}

The current work shows that $\mathrm{CHOL}$ has a significant influence on the buckle delamination processes in physisorbed polymer-tethered membranes containing phospholipids, PEG lipopolymers and CHOL. In the absence of CHOL and at low mol \% PEG lipopolymers, membrane buckling caused by lipopolymers is very limited. Addition of CHOL substantially alters membrane buckling behavior. Specifically, increasing CHOL concentrations lead to more pronounced buckling and expansion of buckle delamination regions. Interestingly, CHOL is partially depleted in buckling regions, whereas lipopolymers and phospholipids show no obvious phase segregation in the polymer-tethered membrane. Overall, our data indicate that the impact of altering CHOL and lipopolymer concentrations in physisorbed polymer-tethered membranes is qualitatively similar, because both affect membrane buckling by changing lateral membrane stress through regulation of repulsive polymer-polymer interactions of lipopolymers. Consequently, no membrane buckling can be observed in phospholipid-CHOL mixtures without lipopolymers. Our results are significant, because CHOL plays an important functional role in biological membranes and because lipopolymers can mimic conditions of molecular crowding caused by proteins on cell surfaces.

\section{Acknowledgments}

This work was supported in part by the National Science Foundation (grants: No. 0920134 and No. 1006552), the IUPUI Nano scale imaging Centre and the IUPUI integrative Nano systems Development Institute.

\section{References}

1. Croll, J.G.A. A new hypothesis for the development of blisters in asphalt pavements. Int. J. Pavement Eng. 2007, 9, 59-67.

2. Pangule, R.C.; Banerjee, I.; Sharma, A. Adhesion induced mesoscale instability patterns in thin pdms-metal bilayers. J. Chem. Phys 2008, 128, 234708:1-234708:6.

3. Mei, H.; Huang, R.; Chung, J.U.; Stafford, C.M.; Yu, H. Buckling modes of elastic thin films on elastic substrates. Appl. Phys. Lett. 2007, 90, 151902:1-151902:3.

4. Audoly, B. Stability of straight delamination blisters. Phys. Rev. Lett. 1999, 83, 4124-4127.

5. Hutchinson, J.W.; Suo, Z. Mixed mode cracking in layered materials. Adv. Appl. Mech. 1992, 29, 63-191.

6. Wirtz, H.R.; Dobbs, L.G. Calcium mobilization and exocytosis after one mechanical stretch of lung epithelial cells. Science 1990, 250, 1266-1269.

7. Longo, M.; Bisagno, A.; Zasadzinski, J.; Bruni, R.; Waring, A. A function of lung surfactant protein sp-b. Science 1993, 261, 453-456. 
8. Alonso, C.; Alig, T.; Yoon, J.; Bringezu, F.; Warriner, H.; Zasadzinski, J.A. More than a monolayer: Relating lung surfactant structure and mechanics to composition. Biophys. J. 2004, $87,4188-4202$.

9. de la Serna, J.B.; Orädd, G.; Bagatolli, L.A.; Simonsen, A.C.; Marsh, D.; Lindblom, G.; Perez-Gil, J. Segregated phases in pulmonary surfactant membranes do not show coexistence of lipid populations with differentiated dynamic properties. Biophys. J. 2009, 97, 1381-1389.

10. Hallett, M.B.; Dewitt, S. Ironing out the wrinkles of neutrophil phagocytosis. Trends Cell Biol. 2007, 17, 209-214.

11. Sackmann, E. Membrane bending energy concept of vesicle- and cell-shapes and shape-transitions. FEBS Lett. 1994, 346, 3-16.

12. Ma, G.; Allen, H.C. New insights into lung surfactant monolayers using vibrational sum frequency generation spectroscopy. Photochem. Photobiol. 2006, 82, 1517-1529.

13. Nakahara, H.; Lee, S.; Shibata, O. Pulmonary surfactant model systems catch the specific interaction of an amphiphilic peptide with anionic phospholipid. Biophys. J. 2009, 96, 1415-1429.

14. Holten-Andersen, N.; Henderson, J.M.; Walther, F.J.; Waring, A.J.; Ruchala, P.; Notter, R.H.; Lee, K.Y.C. K14 peptide induces reversible collapse structures on multiple length scales in model lung surfactant. Biophys. J. 2011, 101, 2957-2965.

15. Siegel, A.P.; Murcia, M.J.; Johnson, M.; Reif, M.; Jordan, R.; Ruhe, J.; Naumann, C.A. Compartmentalizing a lipid bilayer by tuning lateral stress in a physisorbed polymer-tethered membrane. Soft Matter 2010, 6, 2723-2732.

16. Siegel, A.P.; Hussain, N.F.; Johnson, M.; Naumann, C.A. Metric between buckling structures and elastic properties in physisorbed polymer-tethered lipid monolayers. Soft Matter 2012, 8, 5873-5880.

17. Marsh, D.; Bartucci, R.; Sportelli, L. Lipid membranes with grafted polymers: Physicochemical aspects. Biochim. Biophys. 2003, 1615, 33-59.

18. Rovira-Bru, M.; Thompson, D.H.; Szleifer, I. Size and structure of spontaneously forming liposomes in lipid/peg-lipid mixtures. Biophys. J. 2002, 83, 2419-2439.

19. Deverall, M.A.; Gindl, E.; Sinner, E.K.; Besir, H.; Ruehe, J.; Saxton, M.J.; Naumann, C.A. Membrane lateral mobility obstructed by polymer-tethered lipids studied at the single molecule level. Biophys. J. 2005, 88, 1875-1886.

20. Lin, Y.-H.; Minner, D.E.; Herring, V.L.; Naumann, C.A. Physisorbed polymer-tethered lipid bilayer with lipopolymer gradient. Materials 2012, 5, 2243-2257.

21. Garg, S.; Rühe, J.; Lüdtke, K.; Jordan, R.; Naumann, C.A. Domain registration in raft-mimicking lipid mixtures studied using polymer-tethered lipid bilayers. Biophys. J. 2007, 92, 1263-1270.

22. Ritchie, K.; Shan, X.-Y.; Kondo, J.; Iwasawa, K.; Fujiwara, T.; Kusumi, A. Detection of non-brownian diffusion in the cell membrane in single molecule tracking. Biophys. J. 2005, 88, 2266-2277.

23. Marsh, D. Elastic constants of polymer-grafted lipid membranes. Biophys. J. 2001, 81, 2154-2162.

24. Simson, R.; Wallraff, E.; Faix, J.; Niewöhner, J.; Gerisch, G.; Sackmann, E. Membrane bending modulus and adhesion energy of wild-type and mutant cells of dictyostelium lacking talin or cortexillins. Biophys. J. 1998, 74, 514-522. 
25. Hwang, W.C.; Waugh, R.E. Energy of dissociation of lipid bilayer from the membrane skeleton of red blood cells. Biophys. J. 1997, 72, 2669-2678.

26. de Meyer, F.; Smit, B. Effect of cholesterol on the structure of a phospholipid bilayer. Proc. Natl. Acad. Sci. USA 2009, 106, 3654-3658.

27. Deverall, M.A.; Garg, S.; Lüdtke, K.; Jordan, R.; Rühe, J.; Naumann, C.A. Transbilayer coupling of obstructed lipid diffusion in polymer-tethered phospholipid bilayers. Soft Matter 2008, 4, 1899-1908.

28. Rukmini, R.; Rawat, S.S.; Biswas, S.C.; Chattopadhyay, A. Cholesterol organization in membranes at low concentrations: Effects of curvature stress and membrane thickness. Biophys. J. 2001, 81, 2122-2134.

29. Pan, J.; Mills, T.T.; Tristram-Nagle, S.; Nagle, J.F. Cholesterol perturbs lipid bilayers nonuniversally. Phys. Rev. 2008, 100, 198103:1-198103:4.

30. Song, J.; Waugh, R.E. Bending rigidity of sopc membranes containing cholesterol. Biophys. J. 1993, 64, 1967-1970.

31. Subtil, A.; Gaidarov, I.; Kobylarz, K.; Lampson, M.A.; Keen, J.H.; McGraw, T.E. Acute cholesterol depletion inhibits clathrin-coated pit budding. Proc. Natl. Acad. Sci. USA 1999, 96, 6775-6780.

32. Parton, R.G.; Simons, K. The multiple faces of caveolae. Nat. Rev. Mol. Cell Bio. 2007, 8, 185-194.

33. Thiele, C.; Hannah, M.J.; Fahrenholz, F.; Huttner, W.B. Cholesterol binds to synaptophysin and is required for biogenesis of synaptic vesicles. Nat. Cell Biol. 2000, 2, 42-49.

34. Ledesma, M.D.; Dotti, C.G. Membrane and cytoskeleton dynamics during axonal elongation and stabilization. Int. Rev. Cytol. 2003, 227, 183-219.

35. Michael, S.P. Cell control by membrane-cytoskeleton adhesion. Nat. Rev. Mol. Cell Bio. 2001, 2, 392-396.

36. Sun, M.; Northup, N.; Marga, F.; Huber, T.; Byfield, F.J.; Levitan, I.; Forgacs, G. The effect of cellular cholesterol on membrane-cytoskeleton adhesion. J. Cell Sci. 2007, 120, 2223-2231.

37. de la Serna, J.B.; Perez-Gil, J.; Simonsen, A.C.; Bagatolli, L.A. Cholesterol rules: Direct observation of the coexistence of two fluid phases in native pulmonary surfactant membranes at physiological temperatures. J. Biol. Chem. 2004, 279, 40715-40722.

38. Stachowiak, J.C.; Hayden, C.C.; Sasaki, D.Y. Steric confinement of proteins on lipid membranes can drive curvature and tubulation. Proc. Natl. Acad. Sci. USA 2010, 107, 7781-7786.

39. Harvey, T.M.; Jennifer, L.G. Membrane curvature and mechanisms of dynamic cell membrane remodelling. Nature 2005, 438, 590-596.

(C) 2013 by the authors; licensee MDPI, Basel, Switzerland. This article is an open access article distributed under the terms and conditions of the Creative Commons Attribution license (http://creativecommons.org/licenses/by/3.0/). 